\title{
Advanced analysis and event reconstruction for the CTA Observatory
}

\author{
Y. Becherini* ${ }^{* \dagger}$, B. Khélifi ${ }^{\dagger}$, S. Pita* and M. Punch* for the CTA consortium \\ ${ }^{*}$ Astroparticule et Cosmologie, CNRS/Univ. Paris 7, Bât. Condorcet, Paris 75205 cedex 13, France \\ ${ }^{\dagger}$ Laboratoire Leprince-Ringuet, Ecole Polytechnique, CNRS/IN2P3, F-91128 Palaiseau, France
}

\begin{abstract}
.
The planned Cherenkov Telescope Array (CTA) is a future observatory for very-high-energy (VHE) gamma-ray astronomy composed of one site per hemisphere [1]. It aims at 10 times better sensitivity, a better angular resolution and wider energy coverage than current installations such as H.E.S.S., MAGIC and VERITAS.

In order to achieve this level of performance, both the design of the telescopes and the analysis algorithms are being studied and optimized within the CTA Monte-Carlo working group. Here, we present ongoing work on the data analysis for both the event reconstruction (energy, direction) and gamma/hadron separation, carried out within the HAP (H.E.S.S. Analysis Package) software framework of the H.E.S.S. collaboration, for this initial study. The event reconstruction uses both Hillas-parameter-based algorithms and an improved version of the 3D-Model algorithm [2]. For the gamma/hadron discrimination, original and robust discriminant variables are used and treated with Boosted Decision Trees (BDTs) in the TMVA [3] (Toolkit for Multivariate Data Analysis) framework. With this advanced analysis, known as Paris-MVA [4], the sensitivity is improved by a factor of $\sim 2$ in the core range of CTA relative to the standard analyses.

Here we present the algorithms used for the reconstruction and discrimination, together with the resulting performance characteristics, with good confidence, since the method has been successfully applied for H.E.S.S.
\end{abstract}

Keywords: Astronomical observations: gamma-ray; Observation and data reduction techniques; computer modeling and simulation; Mathematical procedures and computer techniques

PACS: $95.85 . \mathrm{Pw}$, 95.75.-z, 95.75.Pq

\section{PARIS-MVA ADVANCED ANALYSIS}

\section{General Description}

The Paris-MVA advanced analysis method is based on a combination of both alreadyknown and newly-derived parameters, combined in an Multi-Variate analysis (MVA). Details can be found in [4] \& references therein.

A total of eight parameters are used as input. The first two are standard "Hillas" image moment-based parameters: Mean-scaled width and Mean-scaled length. The 3DModel [2] provides three further event parameters which are powerful discriminants: 3D-Reduced-Width, its error as given by the likelihood maximization, and the fitted Depth of Shower Maximum. Finally three newly-defined parameters have been developed, which are described below and in [4]. All these parameters are input to the ROOT software suite's TMVA framework [3], applying an MVA based on Boosted Decision Trees (BDTs). Careful testing of the decision tree design (depth, pruning etc.) was car- 
ried out, for example to avoid over-training. In addition, it should be noted that the input parameters were chosen from a larger set using the tests provided in the TMVA package to avoid correlated parameters, since these reduce the efficiency of the BDT procedure.

The training of the BDTs was carried out in a large number of bins (27): over a wide range in Energy, for nine bins in a related 3D-model parameter, $N_{\text {phot }}$, which provides an estimate of the number of Cherenkov photons emitted by the shower; and for three classes in event multiplicity $(2,3-5$, and 6-60 telescopes events) independently of telescope type.

The BDT procedure combines the parameters for each bin in an optimal manner, to provide a single output BDT-parameter on which to cut. Note that the relative efficiency of different parameters varies widely, especially as a function of energy. For Paris-MVA, we choose energy-dependent cuts (i.e. in $N_{\text {phot }}$ bins) using pre-defined $\gamma$-ray efficiencies (e.g. being more restrictive at low energies where the background is more difficult to eliminate but the signal $\gamma$-rays are more abundant).

Note that we explicitly chose not to use goodness-of-fit type discriminants, as these require an excellent understanding of detector characteristics for proper application.

\section{Energy Reconstruction (“Oak”)}

Paris-MVA uses a simple, fast energy-reconstruction procedure, baptized "Oak". Monte-Carlo simulations provide charge profiles as a function of impact parameter ( $q$ vs. $R$ ), for given ranges in Energy $E$, and in depth of Shower Maximum $H$. The simulated profiles obtained are first inverted, to obtain profiles of $E$ vs. $q$ for given ranges in $R$; these profiles are stored for the reconstruction phase.

In the energy-reconstruction phase, for a given telescope, the reconstructed $H$ and $R$ (determined from the Hillas parameters) allow to choose which histograms in $R$ and which bins in $q$ enter into the interpolation (using a weighted tree) for the energy $E_{\text {tel }}$ estimated based on that telescope's information. In the final step, the energies of all the telescopes implicated in an event are combined in a sum weighted by each telescope's charge, giving the estimate of the $\gamma$-ray energy.

For Paris-MVA in CTA, a linear correction with $\log (E)$ (determined from the MonteCarlo simulations) is made in order to take out residual biases, for comparative plots with other analysis techniques. Note that for operational detectors, the standard spectral determination techniques take into account and correct for these biases, allowing to plot fitted spectra versus the true photon energy. The results for example at $1 \mathrm{TeV}$ show an energy resolution of $13 \%$ with $\sim 0 \%$ bias being achieved.

\section{Newly-Developed Parameters}

The three newly-developed parameters input to the BDT, described in [4], are based on the exploitation of the differences in shower development for signal $\gamma$-rays versus the hadronic background. These give, for example, incoherences in the 3D-model (mis-)fit of a hadronic shower with a gamma-ray model. The images (pixel values) predicted in 
each camera by the 3D-model minimization can be used as the basis for a new set of Hillas parameters (HillasOnModel) which are then used to define the new discriminant parameters.

The first new discriminant parameter, $\Omega$, is defined as the angle between the shower direction determined from the original Hillas parameters, and that determined from the HillasOnModel parameters. Two further sensitive discriminant parameters can be defined, based on the Oak energy reconstruction algorithm. By reversing the Oak procedure, given the energy determined from Oak, we can "predict" the charge seen in each telescope, so giving $\Delta Q$, the sum of the squared difference between the observed and predicted charges, divided by the summed observed charges. Finally, we can define $R_{E}$ : the ratio of the energy as determined from the initial Hillas parameters to that found from HillasOnModel. These three new parameters have been shown for H.E.S.S. to provide a gain of $20 \%$ in background rejection for fixed $\gamma$-ray efficiency.

\section{ADAPTATION FOR CTA, SIMULATIONS, \& BDT TRAINING}

Some slight modifications of the 3D-model fitting procedure have been implemented. For the initial condition to the fitting procedure, we use length, width, and shower maximum estimations based only on largest contiguous cluster in each telescope (after standard image cleaning). Also, the information from a telescope is used in the 3Dfit only if it has a total charge $>30$ photoelectrons and with at most 3 clusters of contiguous pixels after standard image cleaning. These modifications allow to adapt the fit convergence for the largest, high-multiplicity events seen with CTA.

All the results were obtained using simulations from the SimTelArray package [5], at $20^{\circ}$ from Zenith, with $\gamma$-rays simulated on-axis between $3 \mathrm{GeV}-300 \mathrm{TeV}$, diffuse electrons in the same energy range, and diffuse protons between $5 \mathrm{GeV}-500 \mathrm{TeV}$.

\section{RESULTS FOR CTA}

The effective area after Paris-MVA BDT cuts and energy-dependent $\theta^{2}$ cut is shown in fig. 1(a), for $\gamma$-rays, and for the electron and proton background. For the point-source $\gamma$ rays, the angular resolution (68\% containment radius) as a function of energy is shown in fig. 1(b).

For the sensitivity calculation shown here, the proton background is scaled by a factor of 1.1 to take into account the presence of heavier species in the charged cosmic-ray flux, and proton and electron background spectra are taken into account. The performance of CTA is shown in fig. 1(c), as applied to the example array "I" (see [1]), an array consisting of 77 telescopes (3 large size telescopes, $24 \mathrm{~m}$ diameter, $5^{\circ} \mathrm{FoV} ; 18$ mid-size, $\sim 12 \mathrm{~m}, 8^{\circ}$; and 56 small-size $7.4 \mathrm{~m}, 10^{\circ}$ ), which is optimized for a good wide-energyrange performance. The figure shows the differential point-source sensitivity in energy bins ( 5 bins per decade, requirement of $5 \sigma$ per bin) for $50 \mathrm{~h}$ observation, for the ParisMVA analysis as compared with that derived from a standard Hillas analysis (see [1]). 

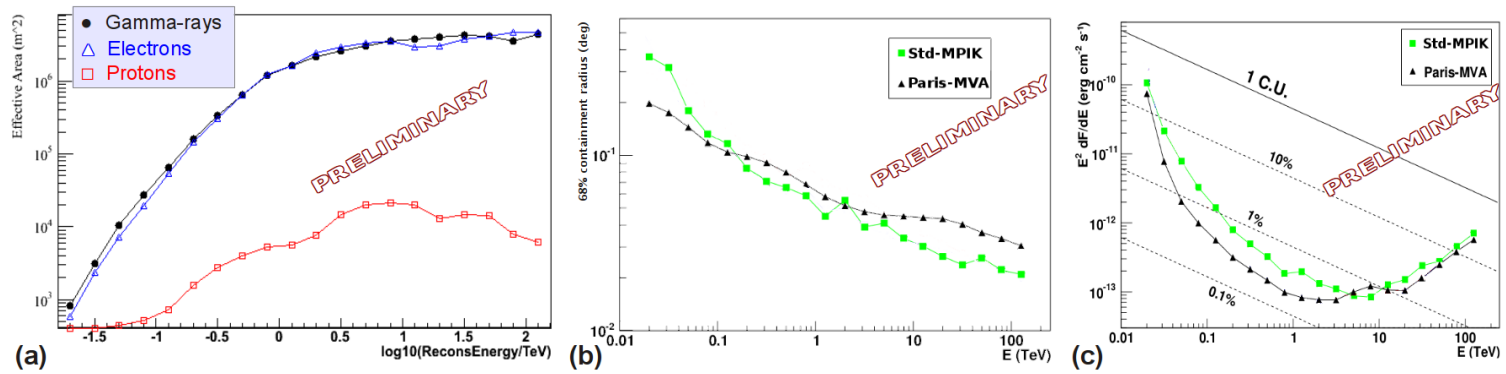

FIGURE 1. (a) Effective collection areas after Paris-MVA and energy-dependent point source $\theta^{2}$ cut; (b) Angular resolution (68\% containment radius) as function of Energy, for point source $\gamma$-rays after ParisMVA cuts, compared with the standard analysis. (c) Differential sensitivity for a point-source as calculated for array "I", for Paris-MVA vs. standard analysis.

\section{CONCLUSIONS}

Paris-MVA was conceived to be flexible, and was implemented within a standard TMVA environment; these features enabled it to be rather quickly adapted from H.E.S.S. to be applied to CTA.

Some modifications have been made to the initialization of the fit in the 3D-model fitting procedure to achieve reasonable convergence at the highest energies (difficult to reach with H.E.S.S. with high statistics), though further work still needs to be done, perhaps to the 3D-model itself (e.g., to take into account the instrument's integration window).

It is readily apparent that the application of the advanced Paris-MVA analysis technique, combining within the MVA framework both previously-known and newly-defined sensitive discrimination variables, allows the performance of the CTA observatory to be improved by a factor $\sim 2$ over the standard analysis.

Comparative studies of the performance under different array layouts and with the different electronics concepts have begun, and should allow the future CTA array to be optimized [6] taking into account such advanced analyses.

\section{REFERENCES}

1. The CTA Consortium, "CTA Design Concepts”, Exp. Astron. 32 (2011) 193-316

2. M. Lemoine-Goumard, B. Degrange, M. Tluczykont, Astropart.Phys. 25 (2006) 195-211

3. http://tmva.sourceforge. net/

4. Y. Becherini et al., Astropart. Phys. 3412 (2011) 858-870

5. K. Bernlöhr, Astropart. Phys. 30 (2008) 149

6. V. Stamatescu, et al. "Towards an optimized design for Cherenkov Telescope Array", this conference 